\title{
An Electrical Burn Resulted from Acupuncture: treatment with V-Y flap combined with Negative pressure approach: A case study
}

\section{Ping Zhou, MM ${ }^{1}$, Xu-Lin Chen, $\mathrm{MD}^{2}$, Fei Wang, $\mathrm{MD}^{3 *}$}

${ }^{1}$ Resident, Department of Burns, the First Affiliated Hospital of Anhui Medical University, Hefei, China.

${ }^{2}$ Chief physicians, Department of Burns, the First Affiliated Hospital of Anhui Medical University, Hefei, China.

${ }^{3}$ Associate chief physicians, Department of Burns, the First Affiliated Hospital of Anhui Medical University, Hefei, China.

* Corresponding Author: Fei Wang, MD, Associate chief physicians, Department of Burns, the First Affiliated Hospital of Anhui Medical University, Hefei, China.

Received date: 10 January 2022; Accepted date: 18 January 2022; Published date: 27 January 2022

Citation: Zhou P, Chen XL, Wang F (2022) An Electrical Burn Resulted from Acupuncture: treatment with V-Y flap combined with Negative pressure approach: A case study. J Med Case Rep Case Series 3(01): https://doi.org/10.38207/JMCRCS/2022/JAN030103

Copyright: (C) 2022 Fei Wang MD. This is an open-access article distributed under the terms of the Creative Commons Attribution License, which permits unrestricted use, distribution, and reproduction in any medium, provided the original author and source are credited.

\begin{abstract}
Acupuncture is an ancient complementary medicine that is now used all over the world. It is true that acupuncture has a relatively low risk. However, many adverse events have been reported due to inappropriate treatment. We report on a patient with a rare case of electrical burn that occurred after acupuncture. A 59-year-old woman, who received acupuncture treatment because of knee pain caused by rheumatic arthritis, presented with sinus tract formation for more than three months. Acupuncture marks and secretions are visible around the wound. Excision of necrotic tissue to normal tissue then covered with V-Y flap and adopted negative pressure therapy (NPT) on the wound resulted in a successful outcome.
\end{abstract}

Keywords: electrical burn, acupuncture, V-Y flap, negative pressure therapy

\section{Introduction}

With a history of over 2500 years, acupuncture of traditional Chinese medicine has become one of the most popular alternative treatments for a variety of illnesses including rheumatic diseases [1,2]. We

\section{Case report}

We report a 59-year-old woman admitted to our hospital with the formation of sinus tract in left knee for more than 3 months, accompanied by a swollen knee. The patient was diagnosed with rheumatic arthritis a year ago. In order to relieve the pain, the patient underwent electrical acupuncture treatment (Figure 1). After several sessions of treatment, the acupuncture site was burnt.

The area of the wound is about $2 \mathrm{~cm} \times 2 \mathrm{~cm}$, and the depth is about 4 $\mathrm{cm}$. The wound showed a relatively darker appearance accompanied present herein a rare case in a female who developed an electrical burn in the left knee secondary to acupuncture treatment. In addition, the wound repair and reconstructive process are also described.

by obvious acupuncture marks and secretions on the skin (Figure 2). Culture of wound secretion was performed, and no bacteria were observed. Then, the patient underwent the debridement of the wound. Given the defects of the deep soft tissue, the V-Y flap was designed to repair the wound. Negative pressure therapy and continuous irrigation (ciNPT) with saline were applied to the closed incision. Therapy was set at a pressure of $75 \mathrm{~mm} \mathrm{Hg}$ in continuous mode. The wound was almost healed on day 14 (Figure 3-4). 


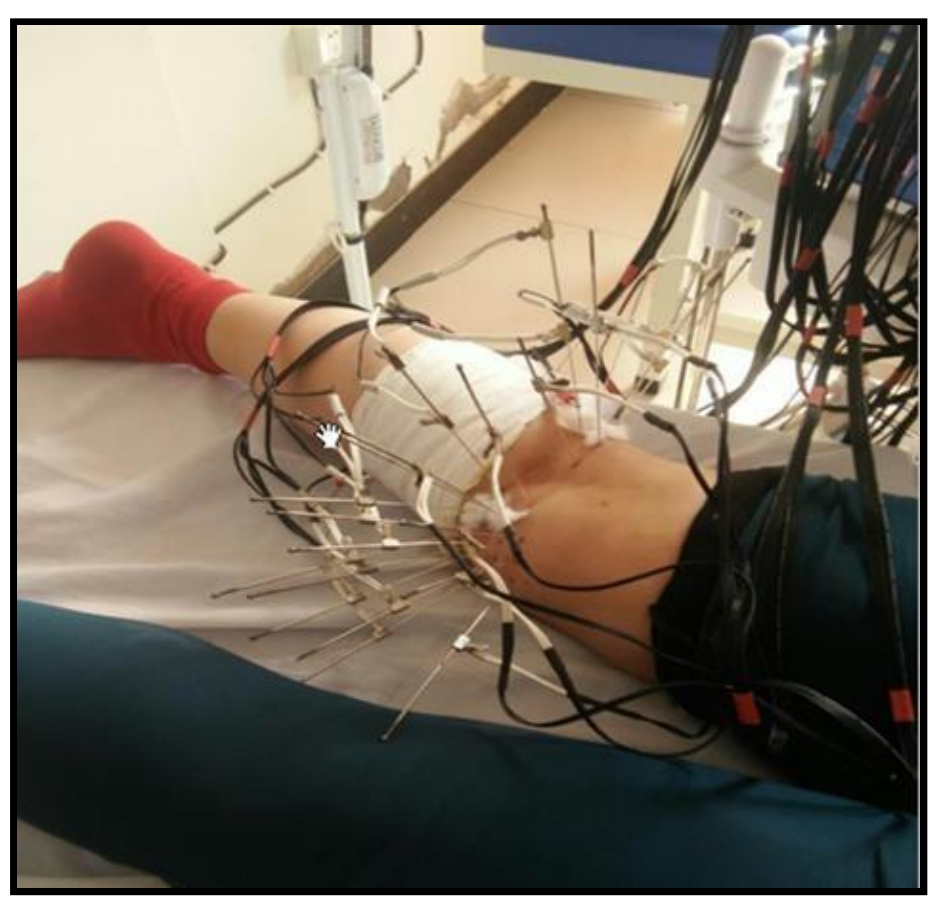

Figure 1: Electrical acupuncture treatment around the knee

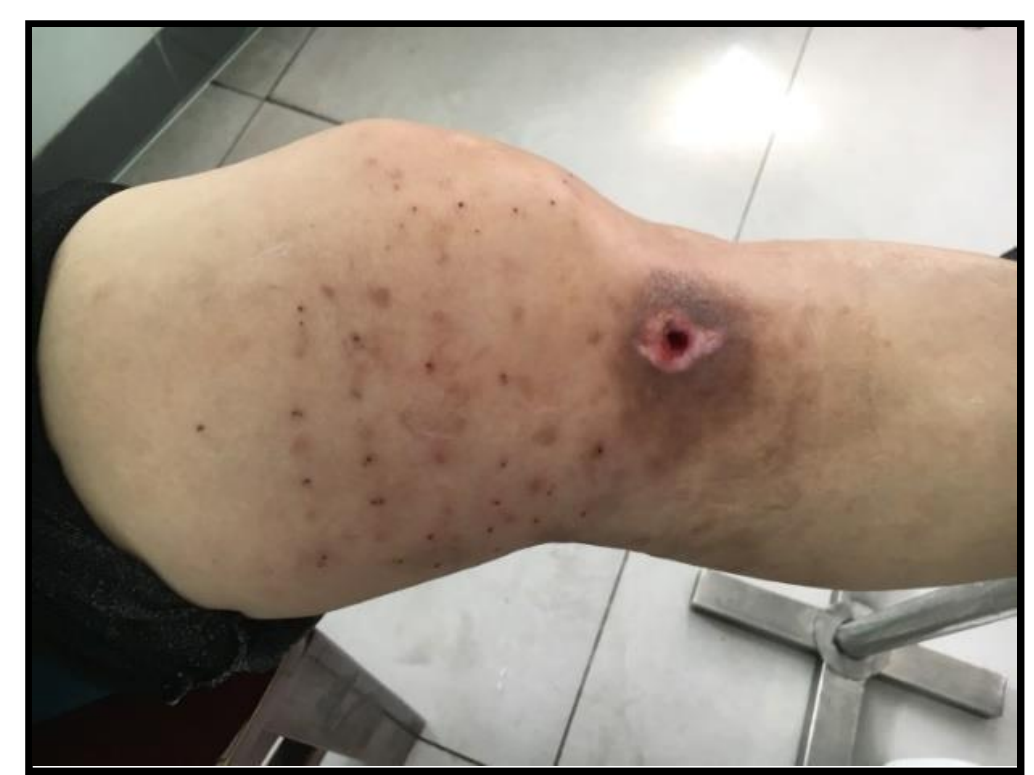

Figure 2: At admission, acupuncture marks were found around the wound. Chronic sinus tract was formed for more than 3 months.
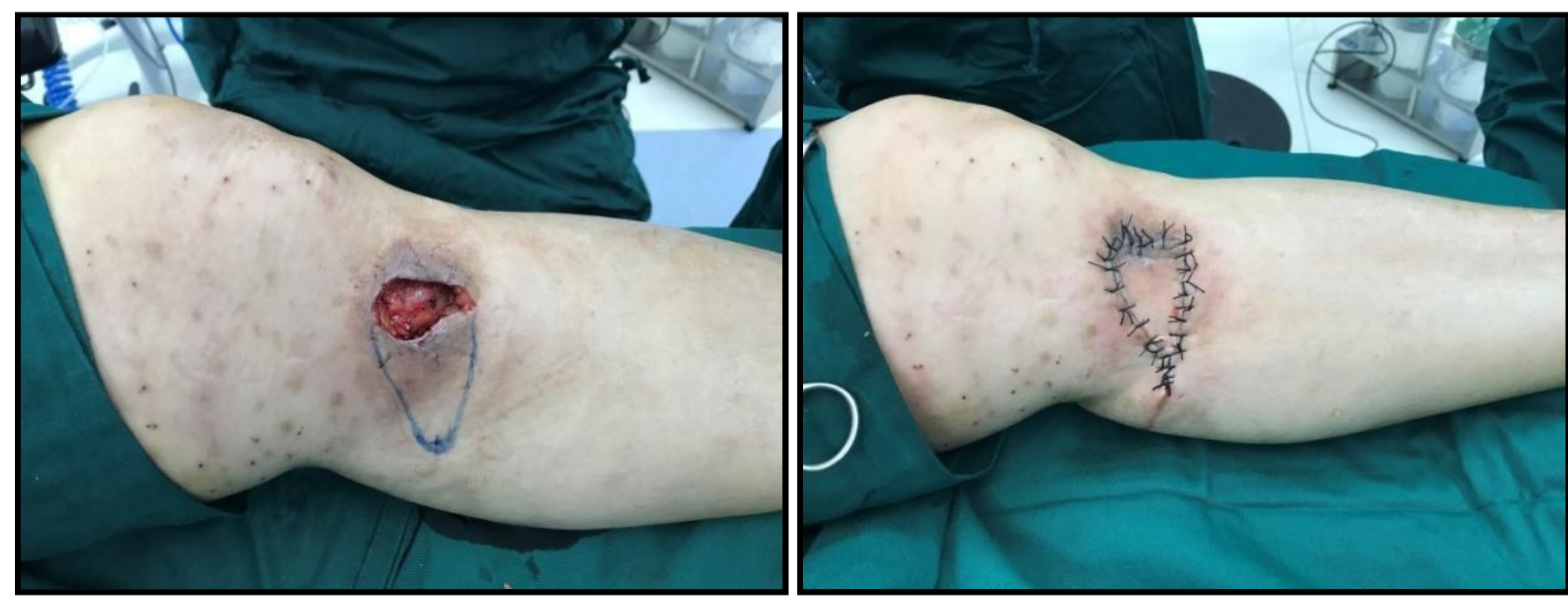

Figure 3: After debridement, the defect was repaired by V-Y flap and immediately covered by ciNPT for 2 weeks.

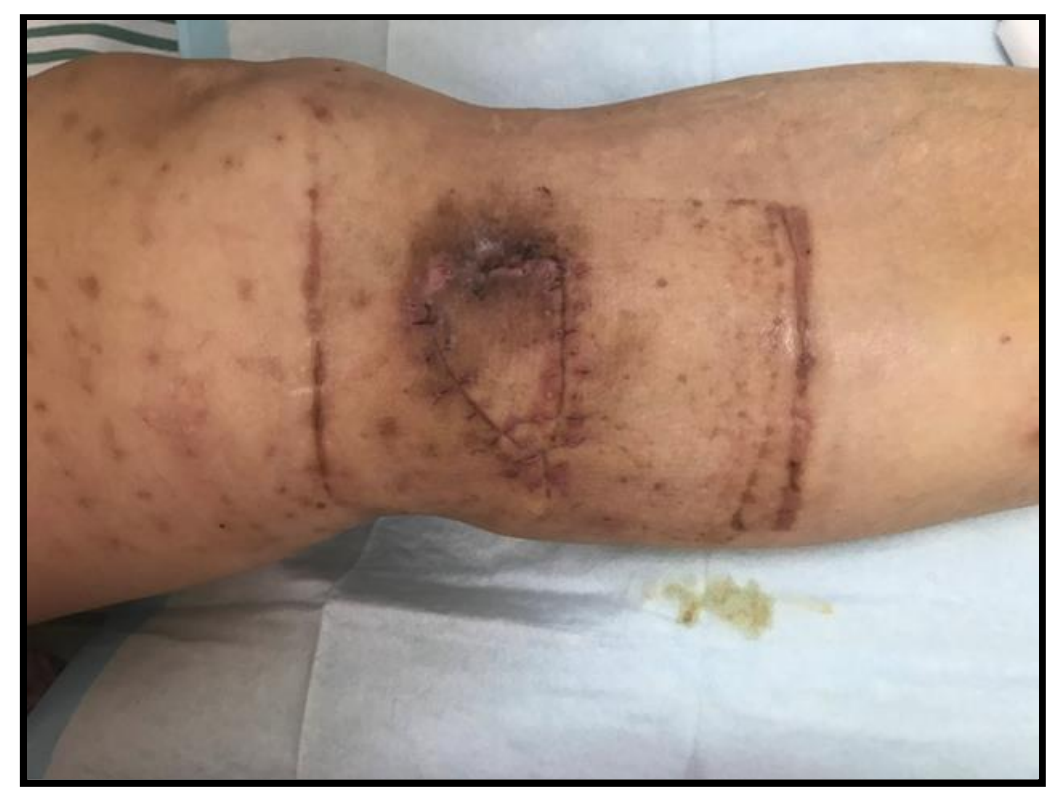

Figure 4: At discharge, chronic wound was well-healed. 


\section{Discussion}

The phenomenon that the patients with rheumatic diseases choose acupuncture as a complementary and alternative treatment not only occurs in Chinese but also in Korea and Israel [3]. When it comes to acupuncture, there is a perception that it is generally safe. Acupuncture could relieve pain, improve quality of life, and cause This case demonstrates the fact that unstable voltage and current of the instrument and insufficient knowledge of acupuncturists result in an electrical burn. The conventional method to treat the wound is to change the dressing regularly, but because of the cost and recurrence, it becomes a major difficulty in clinical treatment. The long course of illness makes the patient painful and affects the quality of life of the patient. It also brings a great burden to the patient mentally and economically.

In this case, we consider that the necrotic tissues should be completely removed. The partial periosteum was exposed, then we designed a V-Y flap to cover the defects. The V-Y advancement flap is a local fasciocutaneous flap that involves mobilizing the adjacent skin and underlying subcutaneous tissue to cover the primary defect. The advancement flap also has the characteristics of small damage, easier operation, ideal appearance, and function. In regard to the chronic wound, negative-pressure wound therapy (NPWT) was immediately used over closed incision after flap reconstruction. NPWT has been demonstrated to initiate a series of biological effects on a closed incision (ciNPT), reducing incision line tension,

\section{References}

1. Seo SY, Lee KB, Shin JS, Lee J, Kim MR, Ha IH, Ko Y, Lee YJ, et al. (2017) Effectiveness of Acupuncture and Electroacupuncture or Chronic Neck Pain: A Systematic Review and Meta-Analysis. Am J Chin Med. 45(8): 1573-1595.

2 Phang JK, Kwan YH, Goh H, Tan VIC, Thumboo J, et al. (2018) Complementary and alternative medicine for rheumatic diseases: A systematic review of randomized controlled trials. Complement Ther Med. 37: 143-157. fewer side effects than other treatment options, for adults with pain. However, many acupuncture-induced adverse events have also been reported which include a spectrum of mild-to-fatal complications such as cardiac tamponade, haemothorax, acute hepatitis B virus infection, bilateral tension pneumothorax [4].

decreasing edema, altering tissue perfusion, and stimulating angiogenesis and the formation of granulation tissue [5]. In this case, CPT was used for 2 weeks and no planned dressing change and resulted in a satisfactory outcome at discharge.

With the increasing use of electrical acupuncture, this case highlights potential injury danger. We herein are supposed to improve the safety awareness of acupuncturists. The following methods, we think, can bring down the incidence of this burn injury significantly. [1] Enhancing responsibility and professional level of acupuncturists to patients. [2] Changing the constant voltage output of electroacupuncture to constant current output, which avoids the damage due to the change of surface resistance value; [5] Improving the level of intelligence of electroacupuncture, so as to more accurately adjust the current of stimulation. Once a wound appears due to complications of acupuncture therapy, surgical intervention in the early stage is of great significance and negative pressure wound therapy alternatively improves the healing on the closed incision.

Conflicts of interest: The authors declare that they have no competing financial interests.

3. Chou PC, Chu HY (2018) Clinical Efficacy of Acupuncture on Rheumatoid Arthritis and Associated Mechanisms: A Systemic Review. Evid Based Complement Alternat Med. 12: 8596918.

4. Mohammad N (2018) Bilateral tension pneumothorax after acupuncture. BMJ Case Rep. 2018: bcr2017221550.

5. Almeida JE, Suárez R, Gibson E (2018)A histological analysis of chronic wounds treated with negative pressure wound therapy to aid healing: a case series. Journal of wound care. 27(1): S28-S34. 